\title{
Correction to: Reply to the letter "Cardiac autonomic evaluation in breast cancer patients: role of cytokines and heart rate recovery"
}

\author{
Claudia Arab ${ }^{1}$ (D) $\cdot$ Luiz Carlos Marques Vanderlei ${ }^{2} \cdot$ Yori Gidron $^{3} \cdot$ Celso Ferreira ${ }^{1}$
}

Published online: 3 July 2018

○) Springer-Verlag GmbH Germany, part of Springer Nature 2018

Correction to: Clinical Research in Cardiology

https://doi.org/10.1007/s00392-018-1292-5

Unfortunately, errors occurred in the original article.

The spelling of the last author's name is "Celso Ferreira".

The affiliation of Yori Gidron is in Belgium.

The original article has been corrected.

The original article can be found online at https://doi.org/10.1007/ s00392-018-1292-5.

Claudia Arab

ac.arabclaudia@gmail.com

1 Departamento de Medicina, Disciplina de Cardiologia,

Hospital São Paulo, Escola Paulista de Medicina,

Universidade Federal de São Paulo, 715 Napoleão de Barros

St, Sao Paulo, SP 04024002, Brazil

2 Departamento de Fisioterapia, Faculdade de Ciência e

Tecnologia, Universidade Estadual Paulista Júlio de Mesquita

Filho, 305 Roberto Símonsen St, Presidente Prudente,

SP 19060-900, Brazil

3 Free University of Brussels (VUB), Boulevard de la Plaine 2, Ixelles 1050, Belgium 